\title{
CORREÇÃO CIRÚRGICA DE ATRESIA ANAL ASSOCIADA À FÍSTULA RETO- VAGINAL EM CADELA DE 4 MESES DE IDADE: RELATO DE CASO
}

\footnotetext{
Paloma Helena Sanches da Silva ${ }_{2}^{1 ;}$ Gabriele Barros Mothé ${ }^{2}$; Solange Cristina Gonçalves da Silva ${ }^{3}$; Nilcéia de Veiga Ramos ${ }^{4}$; Maria de Lourdes Gonçalves Ferreira $^{5}$

${ }^{1}$ Médica veterinária autônoma com Residência na área de Clínica Cirúrgica de Pequenos Animais do programa de Residência em Medicina Veterinária da Universidade Federal Fluminense - UFF, Rio de Janeiro, RJ, Brasil. Autor para correspondência: palomahelena.vet@gmail.com

${ }^{2}$ Residente da área de Clínica Médica de Pequenos Animais do programa de Residência em Medicina Veterinária da Universidade Federal Fluminense - UFF, Niterói, RJ, Brasil.

${ }^{3}$ Mestrando da área de Fisiopatologia e Ciências Cirúrgicas da Universidade Estadual do Rio de Janeiro - UERJ, Rio de Janeiro, RJ, Brasil.

${ }^{4}$ Doutoranda do programa de pós graduação em Medicina Veterinária (Clínica e Reprodução Animal) da Universidade Federal Fluminense - UFF, Niterói, RJ, Brasil.

${ }^{5}$ Docente da Faculdade de Medicina Veterinária do Departamento de Patologia Clínica Veterinária da Universidade Federal Fluminense, Niterói, RJ, Brasil.
}

\section{Recebido em: 03/10/2016 - Aprovado em: 21/11/2016 - Publicado em: 05/12/2016} DOI: 10.18677/EnciBio_2016B_030

\begin{abstract}
RESUMO
As anomalias anorretais são defeitos congênitos de ocorrência rara em diversas espécies; dentre estas, a atresia anal é a mais frequentemente observada na espécie canina e geralmente está associada à fístula reto-vaginal. Nesse trabalho, é descrito o caso de uma cadela, sem raça definida, com 4 meses de idade, atendida no Hospital Universitário de Medicina Veterinária da Universidade Federal Fluminense (HUVET-UFF) com sintomatologia e histórico de eliminação de fezes e urina através de um único orifício, além de prostração, tenesmo e distensão abdominal. Durante os procedimentos diagnóstico e cirúrgico, verificou-se a comunicação entre o reto e a vagina, caracterizando a fístula reto-vaginal secundária à atresia anal. O procedimento cirúrgico é a única possibilidade de sobrevida para pacientes acometidos por esta enfermidade e deve ser realizado o mais precocemente possível. Portanto, esse trabalho objetivou relatar o resultado satisfatório da correção cirúrgica tardia da anomalia em uma cadela de quatro meses, resultando em recuperação da paciente e garantia da qualidade de vida.
\end{abstract}

PALAVRAS-CHAVE: Anoplastia; Cão; Vagina.

\section{SURGICAL CORRECTION OF ATRESIA ANAL ASSOCIATED WITH RECTOVAGINAL FISTULA IN A DOG WITH FOUR MONTH OF AGE: CASE REPORT}

\begin{abstract}
The anorectal malformations are congenital defects of rare occurrence in several species; among these, anal atresia is the most frequently observed in dogs and is usually associated to rectovaginal fistula. This report describes the case of a four
\end{abstract}


months old mixed breed dog, attended at the Veterinary University Hospital of the Universidade Federal Fluminense (HUVET-UFF) with symptoms and history elimination of feces and urine through a single orifice, prostration, tenesmus and abdominal distension. During the diagnostic and surgical procedures, communication between the rectum and vagina was observed, featuring the rectovaginal fistula secondary to anal atresia. The surgical procedure is the only possibility of survival for patients suffering from this disease and should be performed as early as possible. Therefore, this study aimed to report the successful result of surgical correction of the anomaly in a dog with four month age, resulting in recovery of the patient and ensuring the quality of life.

KEYWORDS: Anoplasty; Dog; Vagina.

\section{INTRODUÇÃO}

As anormalidades presentes ao nascimento de um animal, tanto estruturais quanto funcionais, caracterizam-se como anomalias congênitas (ROJAS \& WALKER, 2012; GÓMEZ-CARRILLO et al., 2013; GAZZALA \& MELO, 2016; FERNANDEZ \& HUECK, 2015), que podem ser induzidas por fatores genéticos ou ambientais (LOYNACHAN et al., 2006; GÓMEZ-CARRILLO et al., 2013).

As anomalias congênitas anorretais, como agenesia, hipoplasia e atresia (BANDPEY et al., 2014), são raras em cães e gatos (ELLISON \& PAPAZOGLOU, 2012; GARCÍA-GONZALEZ et al., 2012; DIEZ et al., 2013), mas, dentre estas, a mais frequentemente observada é a atresia anal (retal, anorretal ou ânus imperfurado) cuja abertura do ânus está ausente ou obstruída, estando o reto sem comunicação com o ânus (ELLISON \& PAPAZOGLOU, 2012). Geralmente resulta em megacólon e eliminação anormal das fezes por meio da vagina ou da uretra (VIANNA \& TOBIAS, 2005; RAHAL et al., 2007; ELLISON \& PAPAZOGLOU, 2012).

A prevalência dessa anomalia não está bem estabelecida, mas acontece com mais frequência em cães do que em gatos (GUAIMÁS MOYA et al., 2008) e ocorre durante a embriogênese, por não haver a separação adequada do reto e vagina, gerando a formação de fistula reto-vaginal geralmente associada a atresia anal (MATTHIESEN \& MARRETTA, 2007; ELLISON \& PAPAZOGLOU, 2012; GARCÍAGONZALEZ et al., 2012; AMSTUTZ et al., 2014).

A atresia anal é classificada de acordo com o grau de disgenesia ou agenesia do reto e do ânus, em: Tipo I que consiste num reto normal, mas com o ânus estenótico (estenose anal); Tipo II que é descrito como um reto distal que termina em fundo cego, pois o ânus não é perfurado (ânus imperfurado); Tipo III que consiste em reto normal mas o ânus é coberto por fina camada de pele, formando fundo cego no reto proximal; e, por fim, o Tipo IV onde a comunicação persistente entre o reto e a vagina (fístula reto-vaginal), no caso de fêmeas, ou entre o reto e a uretra (fístula reto-uretral), no caso de machos (VIANNA \& TOBIAS, 2005; ELLISON \& PAPAZOGLOU, 2012).

Sinais de obstrução ou eliminação de fezes através da vulva sugerem sua ocorrência (AMSTUTZ et al., 2014). Outros sinais clínicos incluem principalmente dermatite vulvar, tenesmo, cistite e megacólon (PRASSINOS et al., 2003; VIANNA \& TOBIAS, 2005; MATTHIESEN \& MARRETTA, 2007; RAHAL et al., 2007; ELLISON \& PAPAZOGLOU, 2012; GARCÍA-GONZALEZ et al., 2012).

A incidência desta enfermidade é maior em raças puras, como Spitz, Boston Terrier, Bichon, Maltês, Chow Chow e Schnauzer miniatura, mas também ocorre em animais mestiços (ELLISON \& PAPAZOGLOU, 2012). O diagnóstico baseia-se no 
histórico, sinais clínicos, exame físico e exames complementares, tais como a radiografia contrastada, que revela a extensão do defeito no interior da vagina e confirma a suspeita diagnóstica (MAHLER \& WILLIAMS, 2005; ELLISON \& PAPAZOGLOU, 2012; GARCÍA-GONZALEZ et al., 2012; AMSTUTZ et al., 2014; BRAVO \& TAILLEFER, 2016).

O procedimento cirúrgico e o restabelecimento das estruturas anatômicas normais são contundentes (DOMÍNGUEZ et al., 2012; ELLISON \& PAPAZOGLOU, 2012; GARCÍA-GONZALEZ et al., 2012; AMSTUTZ et al., 2014) e chamados de anoplastia (VIANNA \& TOBIAS, 2005), que pode ser parcial ou completa (MATTHIESEN \& MARRETTA, 2007).

O prognóstico é reservado, pois complicações, como incontinência urinária e fecal (AMSTUTZ et al., 2014) e deiscência de sutura (MATTHIESEN \& MARRETTA, 2007) são frequentes, mas a precocidade do procedimento cirúrgico facilita a recuperação do paciente com menores chances de complicações, reforçando a indicação de tratamento cirúrgico nesses casos (PRASSINOS et al., 2003; ELLISON \& PAPAZOGLOU, 2012; GARCÍA-GONZALEZ et al., 2012).

Esse trabalho objetivou relatar a ocorrência de atresia anal associada à fístula vaginal em uma cadela cuja correção cirúrgica proporcionou completa recuperação da paciente.

\section{RELATO DE CASO}

Uma cadela, sem raça definida e com quatro meses de idade, foi atendida no Hospital Universitário de Medicina Veterinária da Universidade Federal Fluminense (HUVET-UFF), com queixa principal de eliminação de fezes por orifício impróprio, desde o nascimento, além de prostração e distensão abdominal. O exame clínico revelou as seguintes alterações: subdesenvolvimento, escore corporal magro, desidratação de 7\%, mucosas hipocoradas, presença de ectoparasitas (pulgas e carrapatos), ânus imperfurado, abdômen distendido por gases e presença de fezes em vulva (Figura 1).

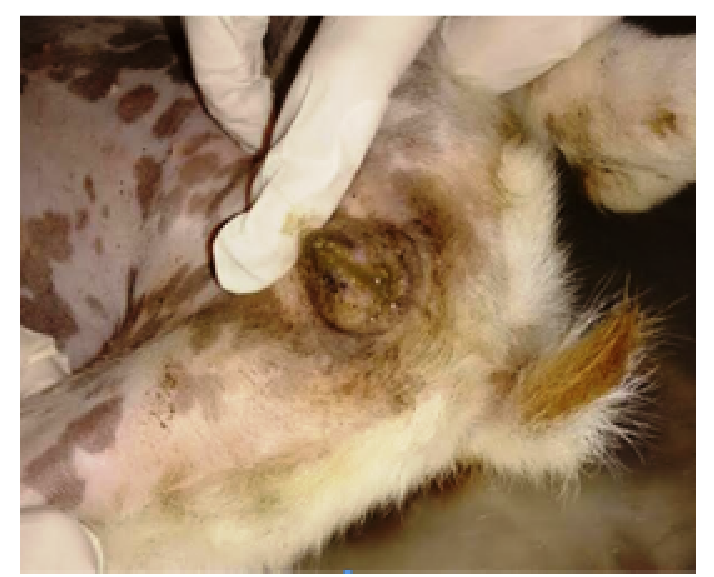

FIGURA 1: Eliminação de conteúdo fecal em região genital da cadela. HUVET-UFF, Niterói, 2015 (Fonte: Arquivo pessoal).

Procedeu-se com exames complementares (hematológico e exames de imagem, como radiografia contrastada com contraste via genital). O hematológico revelou anemia normocítica normocrômica, leucocitose global (de 52.000/ $\mu \mathrm{L}$ ) por 
neutrofilia com desvio nuclear de neutrófilos à esquerda, linfocitose e monocitose, além de diminuição de albumina $(2,0 \mathrm{~g} / \mathrm{dL})$ e cálcio $(8,5 \mathrm{mg} / \mathrm{dL})$, e aumento de fósforo $(8,3 \mathrm{mg} / \mathrm{dL})$.

Inicialmente, foi prescrito metronidazol (20mg/kg, BID, 7 dias), amoxicilina + clavulanato de potássio (20mg/kg, BID, 10 dias), ranitidina (2mg/kg, BID, 10 dias), suplemento com ácido fólico, vitamina B6, vitamina B12, ferro quelatado, DLmetionina, dieta hipercalórica, suplemento de albumina em pó, simeticona e fipronil.

Após 20 dias, a proprietária relatou melhora do quadro clínico, o animal estava mais ativo, sem distensão abdominal evidente e infestação por ectoparasitas. As mucosas encontravam-se normocoradas e não apresentava outras alterações clínicas, além da atresia anal. Foi realizada nova avaliação hematológica, cujos parâmetros haviam melhorado e encontravam-se próximos da normalidade. A radiografia contrastada (Figura 2) foi realizada com sulfato de bário introduzido na vagina e foi de suma importância para o estabelecimento do diagnóstico, indicando a presença de fístula reto-vaginal e megacólon, provavelmente secundários à atresia anal.

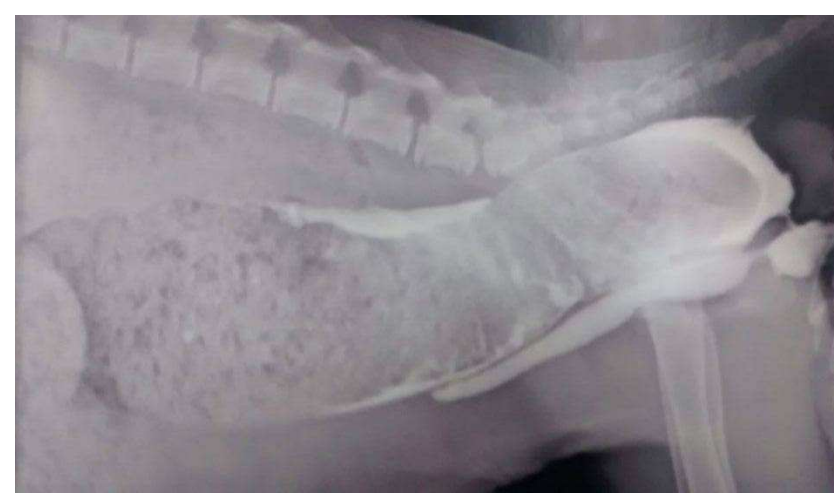
FIGURA 2: Radiografia em posição lateral contrastada de cadela evidenciando presença de fístula reto vaginal e megacólon. HUVET-UFF, Niterói, 2015 (Fonte: Arquivo pessoal).

Nesse caso, optou-se pelo tratamento cirúrgico que consistiu em anoplastia. Para a realização do procedimento cirúrgico, a paciente foi posicionada em decúbito esternal, com a pelve elevada e fixação da cauda para melhor visualização do campo cirúrgico. A cirurgia consistiu em incisão longitudinal na membrana anal (Figura 3A), divulsão do subcutâneo, visualização do reto, seguido pela sua abertura. Foram realizados quatro pontos de reparo com fio nylon 3-0, fixando a parede do reto à pele (Figura $3 \mathrm{~B}$ ) a anoplastia foi concluída empregando o mesmo fio de sutura em padrão interrompido simples (Figura 3C e 3D) em torno de todo o orifício previamente criado. Ao término do procedimento realizou-se o esvaziamento da porção final do cólon e reto por compressão/ massagem abdominal. 


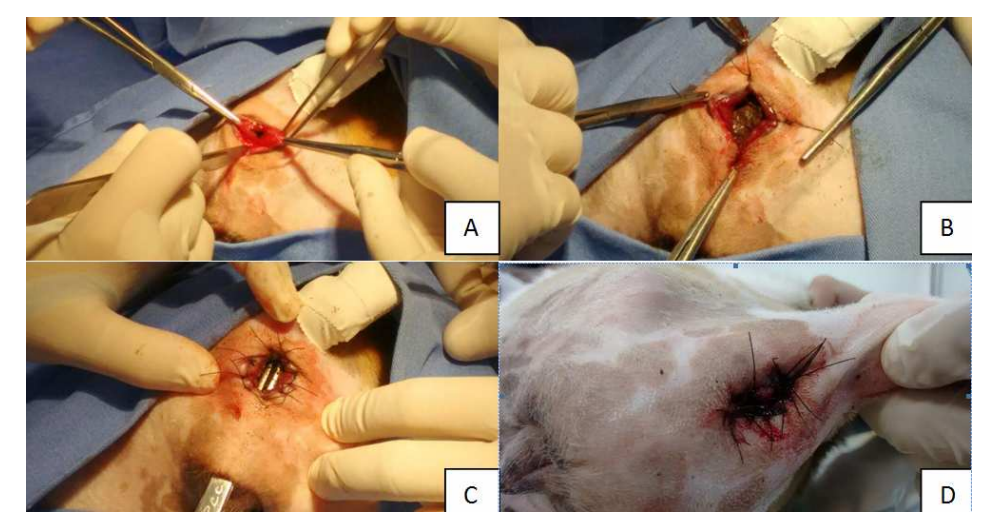

FIGURA 3: Sequência de fotos do procedimento cirúrgico de anoplastia para correção da atresia anal em cão. A) Abertura do ânus após incisão longitudinal na membrana anal e divulsão do tecido subcutâneo para exposição do reto. B) Pontos de reparo em quatro pontos fixando a parede retal à pele, impedindo a retração do reto. C) Orifício previamente criado e demonstração do trajeto fistuloso com o uso de pinça hemostática. D) Reconstrução do ânus. HUVET-UFF, Niterói, 2015 (Fonte: Arquivo pessoal).

O animal foi acompanhado em revisões periódicas após a cirurgia e demonstrou sinais de melhora, recuperando-se clinicamente (Figura 4), com defecação normal, apesar da persistência do megacólon.

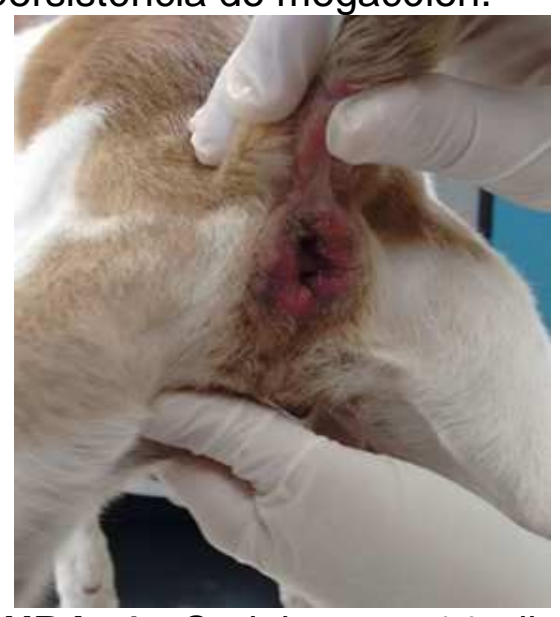

FIGURA 4: Cadela aos 14 dias de pós-operatório de anoplastia, após remoção dos pontos. Nota-se orifício anal em bom estado de cicatrização. HUVET-UFF, Niterói, 2015 (Fonte: Arquivo pessoal).

\section{DISCUSSÃO}

No presente caso, foi registrada fístula reto-vaginal e atresia anal, a qual foi classificada como tipo IV, devido comunicação entre a porção ventral do reto e a parede dorsal da vagina (VIANNA \& TOBIAS, 2005; ELLISON \& PAPAZOGLOU, 2012; AMSTUTZ et al., 2014). 
A sintomatologia dos animais com atresia anal e fístula reto-vaginal consiste em apatia, dilatação abdominal, falha na abertura do ânus e via anormal de eliminação das fezes (PRASSINOS et al., 2003; VIANNA \& TOBIAS, 2005; MATTHIESEN \& MARRETTA, 2007; RAHAL et al., 2007; ELLISON \& PAPAZOGLOU, 2012; GARCÍA-GONZALEZ et al., 2012), mesmos sinais apresentados pela paciente. Tais sinais só foram observados ao desmame, quando o animal passou a ingerir dieta sólida, corroborando com o descrito na literatura, uma vez que, enquanto recém-nato, as mães limpam constantemente os filhotes e a ingesta é apenas líquida, não causando ainda a impactação do cólon e dificultando a observação. Por isso, quando despertam a atenção dos tutores do animal, este já desenvolveu também megacólon (MATTHIESEN \& MARRETTA, 2007), como foi o caso da cadela deste relato, cuja observação dos sintomas foi tardia por parte dos tutores.

Os exames complementares são muito importantes para estabelecer o diagnóstico, principalmente a radiografia contrastada que revela a extensão do defeito (MAHLER \& WILLIAMS, 2005; ELLISON \& PAPAZOGLOU, 2012; GARCÍAGONZALEZ et al., 2012; AMSTUTZ et al., 2014; BRAVO \& TAILLEFER, 2016). Nesse caso, o contraste sulfato de bário foi administrado na vagina da paciente por meio de sonda e foi observada a passagem do contraste positivo para o cólon distal e reto evidenciando a fístula reto-vaginal. O exame radiográfico contrastado foi importante para a confirmação da suspeita clínica, para identificar a posição da fístula (MAHLER \& WILLIAMS, 2005), e para a classificação do tipo de atresia anal, a fim de direcionar o procedimento cirúrgico. Além de julgar a gravidade do caso e o direcionamento clínico, devido a presença de megacólon associado a anomalias congênitas anorretais (BIERY \& SCHWARZ, 2010), guiando assim, a conduta clínica precedente a cirurgia.

Para evitar a ocorrência de megacólon irreversível, além de cistite e agravamento das condições físicas do paciente (PRASSINOS et al., 2003; MATTHIESEN \& MARRETTA, 2007; AMSTUTZ et al., 2014), procedeu-se com a cirurgia corretiva assim que o quadro clínico do paciente foi estabilizado.

A estabilização do paciente com essa deformidade congênita é de extrema importância antes de submetê-lo ao procedimento cirúrgico, uma vez que, por se tratar de um animal muito jovem com sinais clínicos desfavoráveis, o risco de agravamento do quadro durante a cirurgia e no pós-operatório é grande e pode resultar em complicações (ANDRADE et al., 2013).

Nesse caso, o procedimento realizado objetivou corrigir apenas a atresia anal, não interferindo cirurgicamente na fístula reto-vaginal, para permitir o fechamento espontâneo dessa comunicação entre a parede dorsal da vagina e a porção ventral do reto, posteriormente a correção da atresia. Este método foi o de escolha por ser menos invasivo e traumático para a paciente, reduzindo o risco de atingir estruturas adjacentes de importância, como causar algum dano a inervação dessa região (RAHAL et al., 2007). Sendo assim, conforme sugerido na literatura, a intervenção preconizada é a anoplastia e deve ser realizada o mais brevemente possível, assim que estabilizada a condição clínica do paciente que na maioria das vezes encontrase muito debilitado (PRASSINOS et al., 2003; MATTHIESEN \& MARRETTA, 2007).

A cirurgia é a única possibilidade de sobrevida para estes pacientes (GUAIMÁS MOYA et al., 2008; DOMÍNGUEZ et al., 2012; ELLISON \& PAPAZOGLOU, 2012; GARCÍA-GONZALEZ et al., 2012; AMSTUTZ et al., 2014). 
Após a cirurgia, as complicações mais frequentemente relatadas são incontinência fecal, obstipação, tenesmo, constipação, prolapso retal, incontinência urinária, estenose anal e deiscência dos pontos (MAHLER \& WILLIAMS, 2005; VIANNA \& TOBIAS, 2005), neste caso observou-se apenas incontinência fecal inicial.

O tempo em que as fezes ficam retidas e o grau de dilatação do cólon também são critérios prognósticos, sendo necessária a intervenção cirúrgica o mais brevemente possível, para evitar a necessidade de tratamento clínico prolongado, estimulação manual para defecação ou até mesmo colectomia subtotal (PRASSINOS et al., 2003).

A técnica de anoplastia, quando empregada precocemente, mesmo preservando a fístula reto-vaginal até que esta se feche espontaneamente, não têm resultado em complicações (MAHLER \& WILLIAMS, 2005; RAHAL et al., 2007). No caso da cadela citada neste trabalho, mesmo que o diagnóstico e o tratamento tenham sido tardios, a paciente em questão não apresentou grandes complicações pós-operatórias, exceto a incontinência fecal inicial.

\section{CONCLUSÃO}

A correção cirúrgica com emprego de anoplastia, mesmo que tardia, deve ser realizada, pois no paciente relatado o tratamento instituído proporcionou plena recuperação com qualidade de vida.

\section{REFERÊNCIAS}

AMSTUTZ, H. E.; ALLEN, D.G.; ANVERSON, D.P.; JEFFCOTT, L.B.; QUESENBERRY, K.E.; RADOSTITS, O.M.; REEVES,P.T.; WOLF, A.M. Sistema digestive. In: AMSTUTZ, H. E.; ALLEN, D.G.; ANVERSON, D.P.; JEFFCOTT, L.B.; QUESENBERRY, K.E.; RADOSTITS, O.M.; REEVES,P.T.; WOLF, A.M. (Eds) Manual Merk de veterinária. 10 ed. São Paulo: Roca, 2014, p. 127-128.

ANDRADE, A. F.; OLIVEIRA, N. M. S.; MARQUES, M. F. S. Surgical correction of anal atresia and rectovaginal fistula in a puppy: a case report. Acta Veterinaria Brasilica, v. 7, Supl. 1, p. 116-117, 2013. Disponível em: http://periodicos.ufersa.edu.br/revistas/index.php/acta/article/download/3571/5257

BANDPEY, M.L.F.; FLORES, A.M.; MOYA, P.P. El colostograma distal a presión en el manejo radiológico de las malformaciones anorrectales. Revista Cirurgia Pediátrica, v. 27, p. 62-67, 2014. Disponível em: http://www.secipe.org/coldata/upload/revista/2014_27-2_62-67

BIERY, D. N.; SCHWARZ, T. Intestino grosso. In: THRALL, D. E. (Ed). Diagnóstico de Radiologia Veterinária. 5.ed. Rio de janeiro: Elsevier, 2010, p. 792-803.

BRAVO, M.C.B.; TAILLEFER, P.G.H. Qué hacer com El recién nacido obstruído. Radiología, v. 58, supp. 2, p. 70-79, 2016. Disponível em: http://dx.doi.org/10.1016/j.rx.2016.02.005. doi: 10.1016/j.rx.2016.02.005 
DÍEZ, G.O.; RUPÉREZ, J.E.; CARMONA, L.G.; SANTOS, T.T.; BALFAGÓN, C.E. Atresia anal em el perro. Profesión Veterinária, v. 19, n. 81, p. 65-71, 2013. Disponível em: http://www.colvema.org/revista/Colvema81/index.html

DOMINGUÉZ, P.O.; RUIZ, M.M.; HINIESTO, A.S. Resolución quirúrgica de uma atresia anal asociada a uma fistula rectovaginal. Reduca, v. 4, n. 15, p. 132, 2012. Disponível em:

http://www.revistareduca.es/index.php/reduca/article/viewFile/1497/1520

ELLISON, G.W.; PAPAZOGLOU, L.G. Long term results of surgery of atresia ani with or without anogenital malformations in puppies and a kitten: 12 cases (1983-2010). Journal of the American Veterinary Medical Association, v.15, p. 186-192, 2012. Disponível em: http://avmajournals.avma.org/doi/10.2460/javma.240.2.186. doi: 10.2460/javma.240.2.186

FERNANDEZ, J.A.F.; HUECK, L.P. Asociación de fistula rectovaginal congênita com ano normal (Fístulas Tipo $\mathrm{H}$ ) y atresia rectal em uma paciente. Reporte de um caso y breve revisión de La literatura. Revista de Investigação Clínica, v. 56, n. 3, p. 301308, 2015. Disponível em: http://www.redalyc.org/articulo.oa?id=372942435008. id: 372942435008

GARCÍA-GONZÁLEZ, E.M.; ÁNGEL-CARAZA, J.; QUIJANO-HERNÁNDEZ, I.A. Atresia anal em perros y gatos: conceptos actuales a partir de três casos clínicos. Archivos de Medicina Veterinária, v. 44, p. 253-260, 2012. Disponível em: http://dx.doi.org/10.4067/S0301-732X2012000300007. doi: 10.4067/S0301732X2012000300007

GAZZALA, L.P.L.; MELO, F.H.C. Anencefalia e anomalias congênitas: contribuição do patologista ao Poder Judiciário. Revista Bioética, v. 23, n. 3, p. 495-504, 2015. Disponível em: http://dx.doi.org/10.1590/1983-80422015233086. doi: 10.1590/198380422015233086

GÓMEZ-CARRILLO, R.M.V.; ROJAS, A.P.R; GARCÍA, J.T.; BELTRÁN, P.A.M. Atresia anal asociada a fístula rectouretral em oso hormiguero Myrmecophaga tridactyla, em El bioparque lós acarros, villavicencio - Colombia. Conexión Agropecuaria JDC, v. 3, n. 1, p. 77-85, 2013. Disponível em: http://revistasjdc.com/main/index.php/conexagro/article/view/274

GUAIMÁS MOYA, L. E.; AGUILAR, N.; KOSCINCZUK, P. Resolución quirúrgica de una atresia anal asociada a fistula rectovaginal en un cachorro. Revista veterinaria, v. 19, n. 1, p. 46-49, 2008. Disponível em: http://dx.doi.org/10.4067/S0301732X2012000300007. doi: 10.4067/S0301-732X2012000300007

LOYNACHAN, A. T. JACKSON, C.B.; HARRISON, L.R. Complete diphallia, imperforate ani (type 2 atresia ani), and na accessory scrotum in a 5-dayold calf. Journal of Veterinary Diagnostic Investigation, v.18, n.4, p. 408-412, 2006. Disponível em: https://www.ncbi.nlm.nih.gov/pubmed/16921886. PMID: 16921886 
MAHLER, S.; WILLIAN, G. Preservation of the fistula for reconstruction of the anal canal and the anus in atresia and rectovestibular fistula in 2 dogs. Veterinary

Surgery, v. $34, \quad$ p. 148-152, $2005 . \quad$ Disponível em: http://onlinelibrary.wiley.com/doi/10.1111/j.1532-

950X.2005.00024. X/abstract;jsessionid=77DA5921C695ACC0A02F534CE29A42D6.f 01 t03. doi: 10.1111/j.1532-950X.2005.00024.x

MATTHIESEN, D. T.; MARRETA, S. M. Afecções do ânus e reto. In: SLATTER, D. (Ed). Manual de cirurgia de pequenos animais. 3.ed. São Paulo: Manole, 2007. v. 2, cap. 46 , p. $760-780$.

PRASSINOS N. N. PAPAZOGLOU, L.G.; ADAMAMA-MORAITOU, K.K.; GALATOS, A.D.; GOULETSOU, P.; RALLIS,T.S. Congenital anorectal abnormalities in six dogs. The Veterinary Record, v. 153, n. 1, p. 81-85, 2003. Disponível em: http://veterinaryrecord.bmj.com/content/153/3/81 doi: 10.1136/vr.153.3.81

RAHAL, S.C. VICENTE, C.S; MORTARI, A.C; MAMPRIM, M.J.; CAPORALLI, E.H.G. Rectovaginal fistula with anal atresia in 5 dogs. The Canadian Veterinary Journal, v. $48, \quad$ n. $8, \quad$ p. 827-830, 2007. Disponível em: https://www.ncbi.nlm.nih.gov/pmc/articles/PMC1914316/pdf/cvj48pg827.

PMCID:PMC1914316

ROJAS, M.; WALKER, L. Malformaciones congênitas: Aspectos generales y genéticos. International Journal of Morphology, v. 30, n. 4, p. 1256-1265, 2012. Disponível em: http://dx.doi.org/10.4067/S0717-95022012000400003. doi: $10.4067 /$ S0717-95022012000400003

VIANNA, M. L.; TOBIAS, K. M. Atresia ani in the dog: a retrospective study. Journal of the American Animal Hospital Association, v. 41, p. 317-322, 2005. Disponível em: http://jaaha.org/doi/abs/10.5326/0410317. doi: 10.5326/0410317 